\title{
Single molecule evaluation of fluorescent protein photoactivation efficiency using an in vivo nanotemplate
}

\author{
Nela Durisic ${ }^{1}$, Lara Laparra-Cuervo ${ }^{1}$, Ángel Sandoval-Álvarez ${ }^{1}$, Joseph Steven Borbely ${ }^{1}$, \\ Melike Lakadamyali, ${ }^{1, *}$ \\ ${ }^{1}$ ICFO-Institut de Ciències Fotòniques, Mediterranean Technology Park, Castelledefels \\ (Barcelona), Spain \\ *Correspondence should be addressed to melike.lakadamyali@icfo.es
}

\begin{abstract}
Photoswitchable fluorescent probes are central to localization-based super-resolution microscopy. Among these probes, fluorescent proteins are appealing because they are genetically encoded. Moreover, the ability to achieve a one to one labeling ratio between the fluorescent protein and the protein of interest makes them attractive for quantitative single molecule counting. The percentage of fluorescent protein that is photoactivated into a fluorescently detectable form (i.e. photoactivation efficiency) plays a critical role in properly interpreting the quantitative information. It is important to characterize the photoactivation efficiency at the single molecule level to replicate the conditions used in super-resolution imaging. Here, we used the human Glycine receptor expressed in Xenopus oocytes and stepwise photobleaching or single molecule counting-PALM to determine the percentage of photoactivated fluorescent protein for $\mathrm{mEos} 2, \mathrm{mEos} 3.1, \mathrm{mEos} 3.2$, Dendra2, mClavGR2, mMaple, PA-GFP and PA-mCherry. This analysis provides important information that must be considered when using these fluorescent proteins in quantitative super-resolution microscopy.
\end{abstract}

\section{Introduction}

Localization-based super-resolution methods ${ }^{1-3}$ use photoswitchable probes to overcome the diffraction limit. These probes can be activated in sparse numbers over time such that their images are optically resolvable and can be precisely localized ${ }^{4}$. These techniques have been exploited to image sub-cellular structures $^{5-7}$, visualize protein (co)-organization ${ }^{8-10}$ and quantify protein stoichiometry ${ }^{11-15}$.

Probes for localization-based super-resolution imaging ${ }^{16,17}$ typically fall into two categories: (i) reversibly photoswitchable (e.g. Alexa 647, Dronpa, Dreinklang and rsEGFP) ${ }^{3,18-20}$ and (ii) irreversibly photoactivatable and photoconvertible (e.g. PA-GFP, PA-mCherry, mEos2, mEos3.1, mEos3.2, Dendra2, mClavGR2 and mMaple) ${ }^{21-27}$. These different categories provide complementary advantages. For example, probes with many switching cycles lead to smoother images due to repeated sampling of the same structure ${ }^{28}$. The irreversible probes are preferable for protein stoichiometry measurements using single molecule counting with methods like photoactivated localization microcopy (PALM) ${ }^{13},{ }^{14}$, because in principle each probe is detected and counted only once. However, even the irreversible probes can undergo multiple re-activation events ${ }^{29,30}$. Therefore, methods have been developed to reduce over- 
counting artifacts. These include pair correlation analysis (PC-PALM) ${ }^{15}, 31$ or methods that characterize fluorescence off-times and group localizations together based on a characteristic dark time ${ }^{13,30}$. After accounting for re-activation, the remaining localizations are a measure of the number of detected fluorescent probes. To relate this number to biological stoichiometry, one must know the actual percentage of probes that can be photoactivated into a fluorescently detectable form, a property we refer to as the photoactivation efficiency $(P E)$. A fraction of probes may never photoactivate due to several reasons such as premature photobleaching or protonation state of the native form ${ }^{32,33}$. Alternatively, a photoactivated probe may be dark and undetectable. Low photoactivation efficiency can lead to underestimation of protein stoichiometry or protein co-localization ${ }^{34}$.

Photoactivation efficiency has been estimated for some fluorescent proteins using in vitro bulk experiments that follow the evolution of either the absorption spectrum ${ }^{23,33-35}$ or the bands corresponding to the native and activated forms in a protein gel $^{23,33,35}$. However, these experiments may overestimate the photoactivation efficiency since a fraction of the fluorescent protein may be "dark" (undetectable in a single molecule experiment) either in its native form or after photoactivation. Furthermore, the photoactivation efficiency may be different in cells compared to in vitro. We address these issues by characterizing the photoactivation efficiency at the single molecule level inside cells. Our characterization is based on single step photobleaching of labeled-subunits of a nanotemplate (the human Glycine receptor, GlyR), which has a well-defined and stable subunit stoichiometry in Xenopus oocytes ${ }^{36}$. Using this nanotemplate, we characterized the photoactivation efficiency of eight irreversibly switching fluorescent proteins. We further compared the single step photobleaching results with those obtained from single molecule counting-PALM.

\section{Results}

\section{Characterization methods}

\section{Human Glycine receptor as a model system}

We used GlyR as a nanotemplate due to the several advantages it offers. First, it can be expressed in Xenopus oocytes, which do not endogenously express human GlyR ${ }^{36}$. Second, GlyR is incorporated into the oocyte membrane where it is stationary for several minutes ${ }^{36}$, enabling long time measurements even in living cells. Third, in oocytes GlyR has a welldefined and stable stoichiometry in which the heteromers contain $2 \beta: 3 \alpha$ and the homomers contain $5 \alpha$-subunits ${ }^{36,37}$. Therefore, the stoichiometry can be tuned to be two, three or five depending on which subunit is labeled and expressed ${ }^{36}$. Here, we co-express $\beta$ - and $\alpha$ subunits and bias the expression towards heteromers (Methods and ${ }^{36}$ ). Finally, depending on the time after RNA injection, the expression level evolves from single GlyR density to many GlyRs within a diffraction limited volume (Supplementary Fig. 1), and thus the observation time can be tuned to image individual GlyRs. It is important to note that the characterization methods we describe here are not limited to GlyR expressed in Xenopus oocytes, but can be 
easily adapted to any protein complex with a well-defined stoichiometry or tandem repeats of fluorescent proteins expressed in any system (Supplementary Note 1).

\section{Single step photobleaching}

One of the most reliable ways to characterize stoichiometry of small oligomers is to use the stepwise disappearance of fluorescence corresponding to the photobleaching of single molecules ${ }^{36,38,39}$. For example, for a dimer, two photobleaching steps are expected. However, the intensity-time traces typically contain both single and double steps. The single steps are partially due to a small percentage of "missed events" in which two fluorescent proteins photobleach simultaneously ${ }^{36,39}$. These missed events can be accounted for by considering the fluorescence intensity (simultaneous photobleaching of two fluorescent proteins will produce roughly twice the step size compared to a single fluorescent protein) ${ }^{36}$. Often, after correcting for missed events, there is still a number of remaining single step traces, which are due to one of the subunits containing a "dark" fluorescent protein. The percentage of "dark" fluorescent protein can be determined by fitting the counted number of steps to the binomial distribution $^{36,39}$ (Methods). Thus, single step photobleaching not only provides information on protein stoichiometry but also on the probability for a fluorescent protein to be fluorescent. We used this second property to characterize fluorescent protein photoactivation efficiency (Fig. 1a). Since heteromeric GlyRs contain two $\beta$-subunits (or three $\alpha$-subunits), we expected to count a combination of single and double steps (or single, double and triple steps) after photoactivation. Fitting the number of counted steps to the binomial distribution provided the percentage of fluorescent protein that has been successfully photoactivated.

\section{Single molecule counting-PALM}

Single molecule counting-PALM is used to determine protein stoichiometry when single step photobleaching is not feasible (such as high density of proteins) ${ }^{11-14}$. Fluorescent proteins are photoactivated one at a time, detected and subsequently photobleached (Fig. 1b). The number of imaged fluorescent proteins should ideally correspond to the number of actual proteins. For this concept to work, several conditions must be satisfied: (i) fluorescent proteins must be photoactivated slowly to prevent simultaneous activation of multiple molecules within a diffraction limited volume, (ii) all fluorescent proteins must be exhaustively activated and detected during the imaging, (iii) blinking (or re-activation of the same fluorescent protein) must be accounted for and (iv) the photoactivation efficiency must be precisely known.

Imaging schemes have been proposed for controlled photoactivation of fluorescent proteins ${ }^{11,13}$. Furthermore, the cumulative number of fluorescent proteins detected in each frame has been used to determine whether the fluorescent proteins have been exhaustively imaged $^{11}$. The cumulative plot has an initial rise that reaches a plateau once the majority of fluorescent proteins have been detected. Finally, the fluorescence off-times have been used to determine a cut-off time to separate blinking (or re-activation) from photoactivation of a new fluorescent protein ${ }^{13,30}$. Typically, the blinking time is short compared to the photoactivation time, particularly for low protein density and sparse photoactivation regime. Therefore, a plot of fluorescence off-times can be used to set a cut-off time and peaks that appear during 
shorter time intervals can be linked together as belonging to the same fluorescent protein (Fig. 1b). Here, we followed these steps to count the number of $\beta$ - or $\alpha$-subunits in single GlyRs and determined the photoactivation efficiency using this alternative approach.

\section{Photoconvertible fluorescent proteins}

\section{Photoactivation efficiency obtained from single step photobleaching}

mEos 2 is the most commonly used green-to-red converting fluorescent protein in superresolution imaging ${ }^{33}$. Upon illumination with $405 \mathrm{~nm}$ light the fluorescence emission changes from $519 \mathrm{~nm}$ to $584 \mathrm{~nm}^{23}$. Recently, highly monomeric versions of mEos2 have also been generated (mEos3.1 and mEos3.2) ${ }^{25}$. Similarly, mClavGR2 ${ }^{26}, \mathrm{mMaple}^{27}$ and Dendra2 ${ }^{23}$ photoconvert from green to red with $405 \mathrm{~nm}$ light.

We tagged GlyR $\beta$-subunit with one of these fluorescent proteins, co-expressed tagged $\beta$ - and untagged $\alpha$-subunits in Xenopus oocytes and carried out single step photobleaching experiments in living oocytes. We first extracted intensity-time traces from individual GlyRs before photoconversion, which showed stepwise photobleaching (Supplementary Figs. 2a, 3a, 4a and 5a). The counted number of single ( $n=117$ for mEos $2, n=160$ for $\mathrm{mEos} 3.2, n=$ 136 for mClavGR2, $n=121$ for mMaple and $n=144$ for Dendra 2$)$ and double ( $n=210$ for mEos2, $n=149$ for mEos3.2, $n=208$ for mClavGR2, $n=170$ for mMaple and $n=259$ for Dendra2) steps fit well to a binomial distribution given that $\sim 80 \%$ of mEos 2 or Dendra 2 and $\sim 75 \%$ of mClavGR2 or mMaple is fluorescent in their native form in good agreement with other fluorescent protein variants ${ }^{36,39}$ (Table 1). This number was slightly lower for mEos3.2 $(65 \%, n=160$ single and $n=149$ double). The green form of mEos3.1 was less photostable than the other fluorescent proteins, making it hard to determine its photoactivity.

We next determined the in vivo photoconversion kinetics of these fluorescent proteins by continuously illuminating with $405 \mathrm{~nm}$ laser and counting the cumulative number of photoconverted GlyRs in each frame. For example, in the case of mEos2, the cumulative curve reached saturation after $\sim 7$ seconds of illumination with $6.8 \mathrm{~W} / \mathrm{cm}^{2}$ of $405 \mathrm{~nm}$ laser, consistent with the previously determined photoconversion kinetics for this fluorescent $\operatorname{protein}^{25}$ (Supplementary Fig. 2b). Dendra2 photoconversion showed saturation after $\sim 20$ seconds of illumination with $1.5 \mathrm{~W} / \mathrm{cm}^{2}$ of $405 \mathrm{~nm}$ laser (Supplementary Fig. 3b).

We illuminated mEos2 labeled GlyRs with $6.8 \mathrm{~W} / \mathrm{cm}^{2}$ of $405 \mathrm{~nm}$ laser and varied the illumination time between 2-12 seconds to achieve varying levels of photoconversion. For each illumination time, we then imaged the photoconverted molecules with $560 \mathrm{~nm}$ laser until they photobleached (Supplementary Fig. 2c). Despite the fact that under some of these illumination conditions (i.e. $\sim 7$ seconds or longer) the majority of $\mathrm{mEos} 2$ should have photoconverted, the stepwise photobleaching data predominantly contained single steps. Fitting the counted number of single $(n=50)$ and double $(n=33)$ steps to the binomial distribution showed that at most $57 \%$ of mEos 2 was fluorescent in the red form (Table 1 and Fig. 2a). Photoconversion times longer than $\sim 7$ seconds decreased this percentage likely due to photodamage to the red chromophore with extended $405 \mathrm{~nm}$ light illumination (Fig. 2a). It is possible that the incomplete photoconversion is due to the fact that some red mEos2 molecules photobleached during the $405 \mathrm{~nm}$ illumination before we could image them and thus never appeared in the traces. To rule out this possibility, we simultaneously and 
continuously illuminated GlyRs with $405 \mathrm{~nm}$ and $560 \mathrm{~nm}$ lasers and counted steps in the intensity-time traces (Supplementary Fig. 2d). We obtained similar results $(P E=62 \%, n=$ 159 single, $n=132$ double steps) with this approach.

The photoactivation efficiency did not depend on the temperature $\left(60 \%\right.$ at $37^{\circ} \mathrm{C}, n=136$ single and $n=102$ double steps) or $\mathrm{pH}$ of the buffer used for imaging $(62 \%$ at $\mathrm{pH} 5.6 n=83$ single and $n=69$ double steps and $59 \%$ at $\mathrm{pH} 8.5 n=71$ single and $n=52$ double steps), although it is important to mention that the fluorescent protein is intracellular and the local $\mathrm{pH}$ is regulated by the oocyte. Therefore, it is not clear whether changing the extracellular $\mathrm{pH}$ actually changes the $\mathrm{pH}$ experienced by the fluorescent protein. Furthermore, fixation with $1 \%$ PFA did not have a major effect on the photactivation efficiency $(57 \%, n=67$ single and $n=45$ double steps, Supplementary Note 2). Finally, the photoactivation efficiency was independent of the particular subunit that is tagged. Tagging the $\alpha$-subunit gave essentially the same results $(P E=59 \%, n=14$ triple steps, $n=31$ double steps, $n=20$ single steps, fitting extremely well to the binomial distribution for trimeric stoichiometry $\chi^{2}=0.0789, P=$ 0.96) (Fig. 2b).

To determine whether the incomplete photoconversion is due to un-cleaved mEos 2 remaining in the native state or due to a dark population of newly formed mEos2, we checked the photobleaching behavior of the remaining GlyRs in the green channel. Surprisingly, we found that, for short enough illumination times, the remaining percentage of fluorescently detectable green mEos2 when added to red mEos2 brought the total percentage of fluorescently detectable mEos 2 to nearly $100 \%$ (Fig. 2a). Given that only $80 \%$ of mEos 2 is actually fluorescent in its native form, taken together these results indicate that the "dark" native mEos 2 can potentially also photoconvert. Further, the percentage of remaining green mEos 2 reached a plateau at around $37 \%$ and continued illumination with $405 \mathrm{~nm}$ laser did not change this percentage (Fig. 2a). These results indicate that a subpopulation of native mEos2 is unable to photoconvert, which is consistent with previous studies that track the evolution of mEos2 absorption spectrum upon $405 \mathrm{~nm}$ illumination ${ }^{34}$.

We determined the photoactivation efficiency of other photoconvertible fluorescent proteins as $54 \%$ for Dendra2 ( $n=168$ single, $n=100$ double steps, Table 1, Supplementary Fig. 3c and Fig. 2c), 56\% for mClavGR2 $(n=194$ single, $n=121$ double, Table 1, Supplementary Fig. 4b and Fig. 2d) and 51\% for mMaple $(n=427$ single, $n=224$ double steps, Table 1 and Supplementary Fig. 4d). mEos3.2 (Table 1 and Supplementary Fig. 5b) and mEos3.1 had similar photoactivation efficiencies, which were lower than the other fluorescent proteins ( $42 \% n=107$ single, $n=38$ double and $44 \%, n=244$ single, $n=94$ double, respectively).

We also examined the remaining green Dendra2 and mClavGR2 after photoconversion. Dendra2 behaved similar to $\mathrm{mEos} 2$, with the green and red probability adding up to $\sim 100 \%$ and residual green molecules remaining even after extended illumination with $405 \mathrm{~nm}$ laser (Fig. 2c). In the case of mClavGR2, the green and red probabilities only added up to $\sim 84 \%$ and there were very few residual green molecules remaining (Fig. 2d). The low amount of remaining green mClavGR2 indicates either photodamage to the green chromophore with extended $405 \mathrm{~nm}$ illumination before photoconversion could occur or photoconversion of the green chromophore into a fluorescently undetectable form. We are unable to distinguish between these scenarios.

Finally, as a control experiment for the dimeric signal, we also tagged $\alpha 1 \mathrm{E}-\mathrm{Ca}^{+2}$ channel, in which all the subunits are connected into one polypeptide ${ }^{39}$ giving rise to one fluorescent 
protein per channel. As expected, the stepwise photobleaching of the $\alpha 1 \mathrm{E}-\mathrm{Ca}^{+2}$ after photoconversion predominantly ( $96 \%$ ) showed single steps (Supplementary Table 1).

\section{Photoactivation efficiency obtained from single molecule counting-PALM}

For single molecule counting-PALM, we co-expressed photoconvertible fluorescent protein tagged $\beta$ - and mCherry tagged $\alpha$-subunits and lightly fixed the oocytes at a time point of low GlyR density. We first imaged and completely photobleached mCherry and subsequently carried out the PALM experiment. We overlapped the mCherry image with the maximum intensity projection of the PALM time sequence (Fig. 3a). Some GlyRs that appeared in the mCherry image did not have a partner in the PALM image (Fig. 3a). These spots could be due to homomeric GlyRs or GlyRs for which photoconversion completely failed. Similarly, there were some spots which appeared in the PALM image without a partner in the mCherry image (Fig. 3a). These spots could be due to false positives or a new GlyR that became incorporated into the membrane during the PALM imaging since the light fixation did not completely eliminate GlyR transport (Supplementary Fig. 6). Overall, $\sim 60 \%$ of GlyRs that appeared in the mCherry image had a corresponding partner in the PALM image (Fig. 3a). We selected a subset of these GlyRs (30-40\%) for further analysis avoiding ones with elongated point spread function or partially overlapping GlyRs. In the absence of mEos2 there was very little overlap $(<4 \%)$ between the mCherry image and the PALM image, ruling out resurrected mCherry contributing to the signal in PALM (Supplementary Fig. 6).

We first plotted a cumulative curve of the number of molecules detected in each frame of the PALM image (Supplementary Fig. 7a-e), which reached a plateau confirming that we exhaustively imaged the majority of fluorescent proteins. We next plotted intensity-time traces from the PALM time sequence. These traces typically showed single or double "peak clusters" (Fig. 3b-c). The closely spaced peaks in these traces are likely due to the same fluorescent protein which re-appeared multiple times after photoactivation. We refer to this behavior, in which a fluorescent protein returns from a dark state with a short off-time, as "blinking". We determined the blinking kinetics by counting the percentage of molecules that showed blinking and the number of times that each molecule blinked on average (Table 2). We then plotted the distribution of fluorescence off-times in the PALM traces (Supplementary Fig. 7f), which fit well to a single exponential. The short times that dominate the distribution are likely due to blinking whereas the long tail comes from activation of a new fluorescent protein. We set a cut-off time corresponding to the time in which the exponential decayed to $99.8 \%$ of its initial value to account for the majority of blinking events. This cut-off time was 4.1 seconds for $\mathrm{mEos} 2$, and 1.6 seconds for Dendra2, consistent with previous cut-off times used for these fluorescent proteins ${ }^{13}$. The cut-off times for all the fluorescent proteins are summarized in Table 2. We linked together peaks with offtimes smaller than or equal to the cut-off time. After this step, the majority of traces (85-89\%) contained single or double peaks. The small percentage of traces with more than two peaks was partially due to overlapping GlyRs (4\%) and partially due to potential re-activation of the same fluorescent protein after a long off-time $(7-10 \%)^{29}$ (Supplementary Fig. 8). Monomeric $\alpha 1 \mathrm{E}-\mathrm{Ca}^{+2}$ tagged with mEos 2 also contained $\sim 15 \%$ traces with more than one peak (Supplementary Table 1). 
We fit the counted number of single ( $n=83$ for $\operatorname{mEos} 2, n=100$ for $\operatorname{mEos} 3.2, n=117$ for mClavGR2, $n=84$ for mMaple and $n=107$ for Dendra2) and double ( $n=72$ for mEos $2, n=$ 35 for mEos3.2, $n=67$ for mClavGR2, $n=50$ for mMaple and $n=50$ for Dendra2) peaks to the binomial distribution and obtained a similar photoactivation efficiency as determined from single step photobleaching ( $63 \%$ for $\mathrm{mEos} 2,41 \%$ for $\mathrm{mEos} 3.2,53 \%$ for mClavGR2, $54 \%$ for mMaple and $48 \%$ for Dendra2, Table1).

In the above experiments, the activation and excitation light were linearly polarized. Linear polarization can lead to inefficient excitation of the fluorescent proteins if the dipole moment of the fluorescent protein is not properly aligned with the polarization of the exciting light. We believe that laser polarization does not play an important role in these experiments for several reasons (Supplementary Note 2). Nevertheless, to rule out polarization effects, we used a quarter wave plate to change the polarization to circular, re-measured the photoactivation efficiency of mEos 2 and obtained very similar results with both single step photobleaching $(P E=65 \%, n=22$ single and $n=20$ double $)$ and single molecule countingPALM $(P E=60 \%, n=78$ single and $n=59$ double $)$.

\section{Photoactivatable fluorescent proteins}

\section{Photoactivation efficiency determined from single step photobleaching}

PA-GFP is photoactivated from a dark state into bright green fluorescence $(517 \mathrm{~nm})$ upon 405 or $488 \mathrm{~nm}$ light illumination. We tagged the $\beta$-subunit of GlyR with PA-GFP and coexpressed it with mCherry tagged $\alpha$-subunit. The mCherry was used for visualization since PA-GFP is dark before photoactivation. We first examined the photoactivation efficiency after illumination with $488 \mathrm{~nm}$ laser. PA-GFP was photoactivated with high efficiency $(72 \%)$ even with relatively low $488 \mathrm{~nm}$ laser power $\left(7.8 \mathrm{~W} / \mathrm{cm}^{2}\right.$ for 130 seconds) $(n=557$ single and $n=712$ double steps) (Table1). Similarly, illumination with 405 and $488 \mathrm{~nm}$ laser photoactivated $85 \%$ of the PA-GFP ( $n=143$ single and $n=393$ double steps) (Table 1 and Supplementary Fig. 9a). Finally, we co-expressed PA-mCherry tagged $\beta$-subunit together with VFP-tagged $\alpha$-subunit and used the VFP image as a reference to determine the PAmCherry photoactivation efficiency ( $50 \%, n=224$ single and $n=110$ double steps) (Table 1 and Supplementary Fig. 9b).

\section{Photoactivation efficiency determined from single molecule counting-PALM}

For single molecule counting with PA-GFP, we first imaged and photobleached mCherry tagged $\alpha$-subunit and then carried out the PALM experiment. As before, we only used those spots that overlapped with a GlyR in the mCherry image for further analysis. Laser illumination at $488 \mathrm{~nm}$ is needed for imaging PA-GFP but this wavelength also photoactivates PA-GFP, making it impossible to decouple photoactivation from imaging. We therefore started the PALM imaging with $488 \mathrm{~nm}$ laser alone. We used $488 \mathrm{~nm}$ power that was high enough $\left(\sim 162 \mathrm{~W} / \mathrm{cm}^{2}\right)$ to quickly photobleach the PA-GFP molecules once they become photoactivated, typical for PALM. However, illumination with this power rapidly 
and simultaneously photoactivated a high amount of PA-GFP. The cumulative fraction of PA-GFP detected in each frame during PALM showed a sharp rise quickly reaching a plateau, confirming that most of the PA-GFP was photoactivated within the first 10 seconds of PALM imaging (Fig. 4a). Therefore, the likelihood of simultaneously photoactivating both PA-GFPs on the same GlyR was high. Consistent with this observation, we found a low photoactivation efficinecy for PA-GFP $(39 \%, n=99$ single and $n=31$ double peaks $)$ without setting any cut-off time (Fig. 4b). Since single step photobleaching showed high photoactivation efficiency, this result was likely due to both PA-GFPs within the same GlyR being photoactivated simultaneously and thus undercounted.

We also carried out PALM experiments with PA-mCherry tagged $\beta$-subunits, for which VFP tagged $\alpha$-subunit was used as a reference. The intensity time traces mostly showed single or double peaks, with only 15\% of peaks showing blinking on average once (Table 2 and Fig. 4c). Therefore, setting a cut-off time was not necessary for these traces as blinking could easily be distinguished from photoactivation. The PA-mCherry photoactivation efficiency determined in this way was $41 \% \quad(n=60$ single and $n=21$ double peaks $)$. Finally, tagging the $\alpha$-subunit with PA-mCherry, counting the number of traces that contained triple $(n=9)$, double $(n=19)$ and single $(n=22)$ peaks and fitting to the binomial distribution for three subunits gave a probability of $49 \%$ with $\chi^{2}=0.9998(P=0.60)$ for the binomial fit (Fig. 4 d).

\section{Conclusions and Discussion}

In vivo characterization of photoactivation efficiency at the single molecule level carried out in this analysis provides valuable information needed to use these fluorescent proteins in quantitative super-resolution measurements. It is important to emphasize that the single step photobleaching results give a measure of the highest percentage of fluorescent protein that one should expect to detect in PALM imaging. Depending on the experimental conditions, other factors such as background, threshold used for detecting peaks or inability to decouple photoactivation from imaging may further limit the percentage of detected fluorescent protein in PALM (Supplementary Notes 2 and 3). For example, we found that PA-GFP is photoactivated with the highest efficiency. However, high amount of photoactivation with the imaging laser makes it difficult to control activation times, decreasing the detected percentage of fluorescent protein in single molecule counting-PALM. Alternative imaging strategies with very low $488 \mathrm{~nm}$ laser power may be used to overcome this problem at the expense of time needed to photobleach the photoactivated molecules.

We found that a sub-population of native mEos2 and Dendra2 were resistant to photoconversion. It has been suggested that excited-state proton transfer plays an important role in photoconversion ${ }^{33}$ and thus the non-photoconverting molecules may lack the favorable protonation of the native chromophore. Recently, Puchner et al. used tandem repeats of mEos2 fused to the plasma membrane localized Pleckstrin homology (PH) domain of Plc $\delta$ and expressed them at low levels in yeast ${ }^{40}$. Using PALM imaging, blinking correction and binomial statistics, they determined that $\sim 60 \%$ of red mEos 2 was detectable, in excellent agreement with our results, indicating that fluorescent protein photoactivation efficiency is likely independent of the fusion partner and expression system used. mEos2 also had high 
blinking rate, which can be problematic in densely labeled samples since the photoactivation times become shorter obscuring the temporal separation between blinking and photoactivation $^{13}$. Alternative analysis methods that do not rely on defining a cut-off time, such as pair correlation analysis ${ }^{15,31}$, may thus be preferable for quantifying super-resolution data with this fluorescent protein. PA-mCherry and Dendra2 showed moderate photoactivation and lower blinking rates and are therefore well-suited for quantitative superresolution imaging provided that the photoactivation efficiencies are taken into account.

When the stoichiometry is unknown but conserved, in principle it should be possible to distinguish among monomeric, dimeric and trimeric stoichiometry using these fluorescent proteins (Supplementary Fig. 10). While here we focus on single molecule counting, photoactivation efficiency has other important implications. For example, a percentage of the labeled structure (e.g. GlyR) is completely undetected. Finally, photoactivation efficiency also affects quantification of protein co-localization in multi-color experiments.

Acknowledgements: This work was supported in part by Fundació Cellex Barcelona and in part by a Marie Curie International Reintegration grant to M.L. (FP7-PEOPLE-2010-RG). N.D. is a NEST postdoctoral fellow partially supported by the Marie Curie Co-funding of Regional, National and International Programs (COFUND) action of the European Commission. We thank J. Dent, McGill University, for the GlyR- $\alpha$-VFP and GlyR- $\beta$-VFP, X. Zhuang, Harvard University, for mEos2, T. Xu and P. Xu, Chinese Academy of Sciences, for mEos3.1 and mEos3.2, A. McEvoy, University of California, Berkeley, for Dendra2, mClavGR2, mMaple and PA-GFP, T. Misgeld, Technical University of Munich, for PAmCherry and M. Ulbrich, University Freiburg, for $\mathrm{p}^{\mathrm{GEMHE}}-\alpha 1 \mathrm{E}-\mathrm{Ca}^{+2}-\mathrm{mEGFP}$. We thank C. D. Heyes, University of Arkansas, for critical reading of the manuscript, I. Vernos and J. Cela-Gallego, Center for Genomic Regulation, for the oocytes.

Author Contributions: M.L. and N.D designed the experiments. N.D. and L.L.C. performed the experiments and the data analysis. A.S.A provided reagents and performed the cloning of the plasmids. J.S.B. carried out simulations and wrote software. M.L. and N.D. wrote the manuscript. M.L. supervised the project.

Competing Financial Interests: Authors declare no competing financial interests. 


\section{References:}

1. Betzig, E. et al. Imaging intracellular fluorescent proteins at nanometer resolution. Science 313, 1642-1645 (2006).

2. Hess, S.T., Girirajan, T.P. \& Mason, M.D. Ultra-high resolution imaging by fluorescence photoactivation localization microscopy. Biophys J 91, 4258-4272 (2006).

3. Rust, M.J., Bates, M. \& Zhuang, X. Sub-diffraction-limit imaging by stochastic optical reconstruction microscopy (STORM). Nat Methods 3, 793-795 (2006).

4. Huang, B., Babcock, H. \& Zhuang, X. Breaking the diffraction barrier: super-resolution imaging of cells. Cell 143, 1047-1058 (2010).

5. Huang, B., Jones, S.A., Brandenburg, B. \& Zhuang, X. Whole-cell 3D STORM reveals interactions between cellular structures with nanometer-scale resolution. Nat Methods $\mathbf{5}$, 1047-1052 (2008).

6. Lakadamyali, M., Babcock, H., Bates, M., Zhuang, X. \& Lichtman, J. 3D multicolor superresolution imaging offers improved accuracy in neuron tracing. PLoS One 7, e30826 (2012).

7. Balint, S., Verdeny Vilanova, I., Sandoval Alvarez, A. \& Lakadamyali, M. Correlative live-cell and superresolution microscopy reveals cargo transport dynamics at microtubule intersections. Proc Natl Acad Sci U S A 110, 3375-3380 (2013).

8. Kanchanawong, P. et al. Nanoscale architecture of integrin-based cell adhesions. Nature 468, 580-584 (2010).

9. Shroff, H. et al. Dual-color superresolution imaging of genetically expressed probes within individual adhesion complexes. Proc Natl Acad Sci U S A 104, 20308-20313 (2007).

10. Xu, K., Zhong, G. \& Zhuang, X. Actin, spectrin, and associated proteins form a periodic cytoskeletal structure in axons. Science 339, 452-456 (2013).

11. Gunzenhauser, J., Olivier, N., Pengo, T. \& Manley, S. Quantitative super-resolution imaging reveals protein stoichiometry and nanoscale morphology of assembling HIV-Gag virions. Nano Lett 12, 4705-4710 (2012).

12. Lando, D. et al. Quantitative single-molecule microscopy reveals that CENP-A(Cnp1) deposition occurs during G2 in fission yeast. Open Biol 2, 120078 (2012).

13. Lee, S.H., Shin, J.Y., Lee, A. \& Bustamante, C. Counting single photoactivatable fluorescent molecules by photoactivated localization microscopy (PALM). Proc Natl Acad Sci U S A 109, 17436-17441 (2012).

14. Renz, M., Daniels, B.R., Vamosi, G., Arias, I.M. \& Lippincott-Schwartz, J. Plasticity of the asialoglycoprotein receptor deciphered by ensemble FRET imaging and single-molecule counting PALM imaging. Proc Natl Acad Sci U S A 109, E2989-2997 (2012).

15. Sengupta, P. et al. Probing protein heterogeneity in the plasma membrane using PALM and pair correlation analysis. Nat Methods 8, 969-975 (2011).

16. Fernandez-Suarez, M. \& Ting, A.Y. Fluorescent probes for super-resolution imaging in living cells. Nat Rev Mol Cell Biol 9, 929-943 (2008).

17. Lippincott-Schwartz, J. \& Patterson, G.H. Photoactivatable fluorescent proteins for diffraction-limited and super-resolution imaging. Trends Cell Biol 19, 555-565 (2009).

18. Brakemann, T. et al. A reversibly photoswitchable GFP-like protein with fluorescence excitation decoupled from switching. Nat Biotechnol 29, 942-947 (2011).

19. Grotjohann, T. et al. Diffraction-unlimited all-optical imaging and writing with a photochromic GFP. Nature 478, 204-208 (2011).

20. Habuchi, S. et al. Reversible single-molecule photoswitching in the GFP-like fluorescent protein Dronpa. Proc Natl Acad Sci U S A 102, 9511-9516 (2005).

21. Patterson, G.H. \& Lippincott-Schwartz, J. A photoactivatable GFP for selective photolabeling of proteins and cells. Science 297, 1873-1877 (2002).

22. Subach, F.V. et al. Photoactivatable mCherry for high-resolution two-color fluorescence microscopy. Nat Methods 6, 153-159 (2009). 
23. McKinney, S.A., Murphy, C.S., Hazelwood, K.L., Davidson, M.W. \& Looger, L.L. A bright and photostable photoconvertible fluorescent protein. Nat Methods 6, 131-133 (2009).

24. Gurskaya, N.G. et al. Engineering of a monomeric green-to-red photoactivatable fluorescent protein induced by blue light. Nat Biotechnol 24, 461-465 (2006).

25. Zhang, M. et al. Rational design of true monomeric and bright photoactivatable fluorescent proteins. Nat Methods 9, 727-729 (2012).

26. Hoi, $\mathrm{H}$. et al. A monomeric photoconvertible fluorescent protein for imaging of dynamic protein localization. J Mol Biol 401, 776-791 (2010).

27. McEvoy, A.L. et al. mMaple: a photoconvertible fluorescent protein for use in multiple imaging modalities. PLoS One 7, e51314 (2012).

28. Dempsey, G.T., Vaughan, J.C., Chen, K.H., Bates, M. \& Zhuang, X. Evaluation of fluorophores for optimal performance in localization-based super-resolution imaging. Nat Methods $\mathbf{8}$, 1027-1036 (2011).

29. Annibale, P., Scarselli, M., Kodiyan, A. \& Radenovic, A. Photoactivatable Fluorescent Protein mEos2 Displays Repeated Photoactivation after a Long-Lived Dark State in the Red Photoconverted Form. J. Phys. Chem. Lett. 1, 1506-1510 (2010).

30. Annibale, P., Vanni, S., Scarselli, M., Rothlisberger, U. \& Radenovic, A. Quantitative photo activated localization microscopy: unraveling the effects of photoblinking. PLoS One 6, e22678 (2011).

31. Sengupta, P., Jovanovic-Talisman, T. \& Lippincott-Schwartz, J. Quantifying spatial organization in point-localization superresolution images using pair correlation analysis. Nat Protoc 8, 345-354 (2013).

32. Adam, V., Nienhaus, K., Bourgeois, D. \& Nienhaus, G.U. Structural basis of enhanced photoconversion yield in green fluorescent protein-like protein Dendra2. Biochemistry 48, 4905-4915 (2009).

33. Wiedenmann, J. et al. EosFP, a fluorescent marker protein with UV-inducible green-to-red fluorescence conversion. Proc Natl Acad Sci U S A 101, 15905-15910 (2004).

34. Annibale, P., Scarselli, M., Greco, M. \& Radenovic, A. Identification of the factors affecting co-localization precision for quantitative multicolor localization microscopy. Optical Nanoscopy 1:9 (2012).

35. Habuchi, S., Tsutsui, H., Kochaniak, A.B., Miyawaki, A. \& van Oijen, A.M. mKikGR, a monomeric photoswitchable fluorescent protein. PLoS One 3, e3944 (2008).

36. Durisic, N. et al. Stoichiometry of the human glycine receptor revealed by direct subunit counting. J Neurosci 32, 12915-12920 (2012).

37. Lynch, J.W. Native glycine receptor subtypes and their physiological roles. Neuropharmacology 56, 303-309 (2009).

38. Simonson, P.D. et al. Counting bungarotoxin binding sites of nicotinic acetylcholine receptors in mammalian cells with high signal/noise ratios. Biophys J 99, L81-83 (2010).

39. Ulbrich, M.H. \& Isacoff, E.Y. Subunit counting in membrane-bound proteins. Nat Methods 4, 319-321 (2007).

40. Puchner, E.M., Walter, J.M., Kasper, R., Huang, B. \& Lim, W.A. Counting molecules in single organelles with superresolution microscopy allows tracking of the endosome maturation trajectory. Proc Natl Acad Sci U S A (2013). 


\section{Figure Legends:}

Figure 1: Methodology. (a) Single step photobleaching scheme: Fluorescent protein tagged $\beta$-subunit of the human Glycine receptor (GlyR) is co-expressed in Xenopus oocytes with the untagged $\alpha$-subunit to create hetero-pentameric ion channels with two $\beta$-subunits per channel. The fluorescent proteins are photoactivated by varying powers and durations of activation laser (violet arrow). For each power (or illumination time) the photoactivated fluorescent proteins are imaged until they photobleach (green arrow). Intensity-time traces can then be used to count the number of single or double photobleaching steps. Since GlyR has a conserved stoichiometry with two $\beta$-subunits, fitting the number of counted steps to the binomial distribution provides a measure of the percentage of photoactivated fluorescent protein. (b) Single molecule counting-PALM scheme: GlyRs are expressed in Xenopus oocytes as in (a). Fluorescent proteins are photoactivated one at a time by incrementally increasing the photoactivation laser power over time. An imaging laser at high power is used to excite and subsequently photobleach the photoactivated fluorescent protein. Intensity time traces are then used to count the number of peaks. Peaks with short off-times (green arrow and inset) are linked together as one peak. Peaks with long off-times (magenta arrow) are counted as a new photoactivation event. The counted number of peaks is fit to the binomial distribution to calculate the percentage of photoactivated fluorescent protein.

Figure 2: Photoactivation efficiency of mEos2, Dendra2 and mClavGR2 determined from single step photobleaching. (a) Magenta plot shows the effect of varying the $405 \mathrm{~nm}$ laser illumination time on the percentage of photoactivated mEos2. The green curve shows the percentage of remaining native (green) mEos2 after exposure to varying times of $405 \mathrm{~nm}$ laser illumination. (b) Histogram (black) showing the number of counted single $(n=20)$, double $(n=31)$ and triple $(n=14)$ steps in heteromeric channels in which the $\alpha$-subunit was tagged with mEos2. The red histogram is a fit to the binomial distribution for trimeric stoichiometry. The fit gave a photoactivation efficiency of $59 \%$ and goodness of fit of $\chi^{2}=$ 0.0789 or $P=0.96$ (c) Magenta plot shows the effect of varying the $405 \mathrm{~nm}$ laser illumination time on the percentage of photoactivated Dendra2. The green curve shows the percentage of remaining native (green) Dendra2 after exposure to varying times of $405 \mathrm{~nm}$ laser illumination. (d) Magenta plot shows the effect of varying the $405 \mathrm{~nm}$ laser illumination time on the percentage of photoactivated mClavGR2. The green curve shows the percentage of remaining native (green) mClavGR2 after exposure to varying times of $405 \mathrm{~nm}$ laser illumination. The green probability could not be reliably determined after the last point due to very few remaining molecules in the green channel.

Figure 3: Photoactivation efficiency of $\mathrm{mEos} 2$ and Dendra 2 determined from single molecule counting-PALM. (a) Image showing the overlap between mCherry-tagged $\alpha$ subunit (magenta) and the maximum intensity projection of the PALM sequence of mEos2tagged $\beta$-subunit (green). The GlyRs that appear in both channels (arrow) are used for further analysis while the mCherry alone (full arrowhead) and mEos2 alone (empty arrowhead) spots are discarded from the analysis. (b-c) Example intensity-time PALM traces for mEos2 (b) and Dendra2 (c). 
Figure 4: Photoactivation efficiency of PA-GFP and PA-mCherry determined from single molecule counting-PALM. (a) Curve showing the cumulative number of detected PA-GFP molecules per frame during a PALM experiment in which PA-GFP was photoactivated with $162 \mathrm{~W} / \mathrm{cm}^{2}$ of $488 \mathrm{~nm}$ laser illumination alone. The cumulative number has been normalized by the maximum number of counted molecules. (b-c) Example intensity-time PALM trace for PA-GFP (b) and PA-mCherry (c). (d) Histogram (black) showing the number of counted single $(n=22)$, double $(n=19)$ and triple $(n=9)$ peaks in heteromeric channels in which the $\alpha$-subunit was tagged with PA-mCherry. The red histogram is a fit to the binomial distribution for trimeric stoichiometry. The fit gave a photoactivation efficiency of $49 \%$ and goodness of fit of $\chi^{2}=0.9998$ or $P=0.60$. 


\section{Tables}

Table 1: Summary of the photoactivation efficiencies $(P E)$ obtained for different fluorescent proteins using single step photobleaching and single molecule counting-PALM. Where appropriate, the different experiments reported in the text have been combined to report one value (e.g. non-continuous and continuous illumination with $405 \mathrm{~nm}$, with and without quarter wave plate, living and fixed cells, dimeric and trimeric stoichiometry). The uncertainty reported is the standard deviation calculated from different oocytes. The n's reported are the total number of traces analyzed. The data comes from a minimum of two oocytes obtained from different frogs and multiple fields of view per oocyte. Similar results were obtained for mEos3.1 as mEos3.2, which is not shown in the table.

\begin{tabular}{|c|c|c|c|c|c|c|}
\hline & \multicolumn{5}{|c|}{ Single-step photobleaching } & \multirow{2}{*}{\begin{tabular}{|c}
$\begin{array}{c}\text { Single } \\
\text { molecule } \\
\text { counting } \\
\text { PALM }\end{array}$ \\
PE PALM \\
(mean \pm s.d.)
\end{tabular}} \\
\hline $\begin{array}{c}\text { Fluorescent } \\
\text { Protein }\end{array}$ & $\begin{array}{l}\text { Green Form } \\
\text { (mean } \pm \text { s.d.) }\end{array}$ & $\begin{array}{l}P E 405 \mathrm{~nm} \\
(m e a n \pm \text { s.d.) }\end{array}$ & $\begin{array}{l}P E 488 \mathrm{~nm} \\
(\text { mean } \pm \text { s.d. })\end{array}$ & $\begin{array}{c}405 \mathrm{~nm} \\
\text { power } \\
\text { density and } \\
\text { illumination } \\
\text { time }\end{array}$ & \begin{tabular}{|c|}
$488 \mathrm{~nm}$ \\
power \\
density and \\
illumination \\
time
\end{tabular} & \\
\hline mEos2 & $\begin{array}{l}\mathbf{7 8} \pm \mathbf{1 \%} \\
(n=327)\end{array}$ & $\begin{array}{l}\mathbf{6 0} \pm \mathbf{3 \%} \\
(n=1106)\end{array}$ & NA & $\begin{array}{l}6.8 \mathrm{~W} / \mathrm{cm}^{2} \\
\text { for } 6.7 \mathrm{~s}\end{array}$ & NA & $\begin{array}{l}\mathbf{6 1} \pm \mathbf{2 \%} \\
(n=292)\end{array}$ \\
\hline mEos3.2 & $\begin{array}{l}\mathbf{6 5} \pm \mathbf{2 \%} \\
(n=309)\end{array}$ & $\begin{array}{c}\mathbf{4 2 \%} \mathbf{0} \pm \mathbf{9 \%} \\
(n=145)\end{array}$ & NA & $\begin{array}{l}6.5 \mathrm{~W} / \mathrm{cm}^{2} \\
\text { for } 6.3 \mathrm{~s}\end{array}$ & NA & $\begin{array}{l}\mathbf{4 1} \pm \mathbf{4 \%} \% \\
(n=135)\end{array}$ \\
\hline mClavGR2 & $\begin{array}{l}\mathbf{7 5} \pm \mathbf{2 \%} \\
(n=344)\end{array}$ & $\begin{array}{c}\mathbf{5 6 \%} \begin{array}{c}\mathbf{1} \% \\
(n=315)\end{array} \\
\end{array}$ & NA & $\begin{array}{l}6.5 \mathrm{~W} / \mathrm{cm}^{2} \\
\text { for } 15 \mathrm{~s}\end{array}$ & NA & $\begin{array}{l}\mathbf{5 3} \pm \mathbf{8 \%} \\
(n=184)\end{array}$ \\
\hline mMaple & $\begin{array}{l}74 \pm 4 \% \\
(n=291)\end{array}$ & $\begin{array}{c}\mathbf{5 1 \%} \% \mathbf{2 \%} \\
(n=651)\end{array}$ & NA & $\begin{array}{c}3.2 \mathrm{~W} / \mathrm{cm}^{2} \\
\text { for } 3 \mathrm{~s}\end{array}$ & NA & $\begin{array}{l}\mathbf{5 4} \pm 3 \% \\
(n=134)\end{array}$ \\
\hline Dendra2 & $\begin{array}{l}\mathbf{8 2} \pm \mathbf{2 \%} \\
(n=403)\end{array}$ & $\begin{array}{l}\mathbf{5 4} \pm \mathbf{3 \%} \\
(n=268)\end{array}$ & NA & $\begin{array}{c}1.5 \mathrm{~W} / \mathrm{cm}^{2} \\
\text { for } 2.2 \mathrm{~s}\end{array}$ & NA & $\begin{array}{l}\mathbf{4 8} \pm \mathbf{1 \%} \% \\
(n=157)\end{array}$ \\
\hline PA-GFP & NA & $\begin{array}{c}\mathbf{8 5 \%} \pm \mathbf{5 \%} \\
(n=536)\end{array}$ & $\begin{array}{c}\mathbf{7 2 \%} \pm \mathbf{4 \%} \\
(n=1269)\end{array}$ & $\begin{array}{l}0.4 \mathrm{~W} / \mathrm{cm}^{2} \\
\text { for } 140 \mathrm{~s}\end{array}$ & $\begin{array}{l}7.8 \mathrm{~W} / \mathrm{cm}^{2} \\
\text { for } 130 \mathrm{~s}\end{array}$ & $\begin{array}{l}\mathbf{3 9} \pm \mathbf{6 \%} \\
(n=130)\end{array}$ \\
\hline PA-mCherry & NA & $\begin{array}{c}\mathbf{5 0 \%} \pm \mathbf{3 \%} \\
(n=334)\end{array}$ & NA & $\begin{array}{l}6.8 \mathrm{~W} / \mathrm{cm}^{2} \\
\text { for } 2.2 \mathrm{~s}\end{array}$ & NA & $\begin{array}{l}\mathbf{4 5} \pm \mathbf{6 \%} \\
(n=131)\end{array}$ \\
\hline
\end{tabular}


Table 2: Blinking kinetics of and cut-off times used for each fluorescent protein

\begin{tabular}{|c|c|c|c|}
\hline $\begin{array}{c}\text { Fluorescent } \\
\text { Protein }\end{array}$ & $\begin{array}{c}\text { molecules that } \\
\text { blinked (\%) }\end{array}$ & $\begin{array}{c}\text { Average \# of times a molecule } \\
\text { blinked }\end{array}$ & $\begin{array}{c}\text { Cut-off time } \\
\text { (s) }\end{array}$ \\
\hline mEos2 & 58 & 2.8 & 4.1 \\
\hline mEos3.2 & 50 & 2.4 & 3 \\
\hline mClavGR2 & 49 & 3 & 1.9 \\
\hline mMaple & 49 & 3.4 & 2.6 \\
\hline Dendra2 & 30 & 2.7 & 1.6 \\
\hline PA-mCherry & 15 & 1 & NA \\
\hline
\end{tabular}

\section{Online Methods}

\section{Constructs}

Plasmid constructions containing the fusion proteins of glycine receptor $\beta$-subunit with the different fluorescent proteins was performed using the plasmid, pT7 GlyR- $\beta$-VFP. In this plasmid the fluorescent protein (VFP) is attached to the GlyR $\beta$-subunit as an internal fusion taking advantage of a KpnI restriction site naturally present in the DNA coding sequence corresponding to an intracellular loop between two transmembrane domains ${ }^{36}$.

We constructed 8 plasmids each of GlyR $\beta$-subunit with all eight fluorescent proteins (mEos2, mEos3.2, mEos3.1, mClavGR2, mMaple, Dendra2 PA-GFP and PA-mCherry). Original VFP was removed by digesting the corresponding plasmid with KpnI restriction enzyme followed by dephosphorilation using CIAP. mEos2, mEos3.2, mEos3.1, mClavGR2, mMaple, Dendra2, PA-GFP and PA-mCherry fluorescent proteins were inserted in the exact same position. These fluorescent proteins were amplified by PCR from original plasmids in our collection using modified primers (Supplementary Table 2) in which KpnI restriction sites were generated to maintain the reading frame intact through the full length of the fusion proteins. In the case of mEos $2, \operatorname{mEos} 3.1$ and mEos3.2 it was necessary to perform a site directed mutagenesis to remove a natural occurring KpnI (GGTACC) restriction site in its sequence. In this particular case the KpnI restriction site was replaced by a BamHI site (GGATCC). As a consequence of that a T123S mutation in the fluorescent protein was generated. We note that this is a very minor modification since both amino acids have the same polarity, same pKa and almost the same size and only differ by one methyl group $\left(\mathrm{CH}_{3}\right)$ in the side chain of the amino acid (threonine has an extra methyl group). We also obtained similar photoactivation kinetics (see text), blinking kinetics (see text), and photon count distribution (Supplementary Fig. 11) for modified mEos2 to what has previously been reported in the literature for the native $\mathrm{mEos} 2$.

All the plasmids constructed were verified by direct sequencing to ensure the right orientation of the insert, the consistency of the whole sequence of the fragment amplified by PCR and the preservation of the reading frame in the joints in both ends of the fluorescent protein insertion. The GlyR $\alpha$-subunit containing mCherry, mEos 2 and PA-mCherry was generated in a similar way from GlyR- $\alpha$-VFP. 
Cloning was performed to remove the original mEGFP in the $\alpha 1 \mathrm{E}-\mathrm{Ca}^{+2}$ channel $\left(\mathrm{p}^{\mathrm{GEMHE}}-\alpha 1 \mathrm{E}\right.$ $\mathrm{Ca}^{+2}$-mEGFP) and replace it with mEos 2 or PA-mCherry. The fluorescent proteins were amplified using PCR using modified primers with SpeI restriction sites. $\alpha 1 \mathrm{E}-\mathrm{Ca}^{+2}-\mathrm{mEGFP}$ plasmid was digested with this restriction enzyme to remove mEGFP, the digested plasmid was dephoshorilated with CIAP and the SpeI-digested PCR products were ligated. The resulting plasmids were verified by direct sequencing.

\section{Expression in Xenopus oocytes}

The pT7-GlyR cDNA constructs were linearized with BamHI, SmaI and SalI restriction enzymes and capped cRNAs were transcribed using the mMESSAGE mMACHINE®T7 kit (Life Technologies). Synthesized cRNAs were recovered by $\mathrm{LiCl}$ precipitation and resuspended in nuclease-free water at a final concentration of $0.5-1.0 \mu \mathrm{g} / \mu \mathrm{L}$. The pT7-calcium cDNA constructs were linearized with NheI restriction enzyme. The rest of the steps of RNA synthesis were the same as the pT7-GlyR.

Xenopus oocytes were harvested from mature female Xenopus laevis according to standard procedures and maintained at $20^{\circ} \mathrm{C}$ in ND96 solution $(96 \mathrm{mM} \mathrm{NaCl}, 2 \mathrm{mM} \mathrm{KCl}, 1.8 \mathrm{mM}$ $\mathrm{CaCl}_{2}, 1 \mathrm{mM} \mathrm{MgCl} 2,5 \mathrm{mM}$ HEPES, pH 7.5) ${ }^{41}$. Oocytes were injected with $40 \mathrm{~nL}$ of cRNA using the Nanoject system (Drummond Scientific) and FemtoJet system (Eppendorf) and incubated for 12-20 hours prior to photobleaching or PALM experiments. The $\beta$-subunit RNA was injected at a concentration 5-10-fold higher than the $\alpha$-subunit RNA. Since $\beta$ subunits do not form homomeric channels or do not insert into the membrane as monomers, this injection strategy was used to bias the expression towards heteromeric channels ${ }^{36}$. For single step photobleaching experiments, vitelline envelope and extracellular matrix were removed by incubating the oocytes in $0.05 \mathrm{mg} / \mathrm{mL}$ proteinase K (Roche Applied Science) for 5-6 min immediately before the experiment ${ }^{42}$. The oocytes were then allowed to settle on the surface of a chambered coverglass prior to microscopy. For single molecule counting-PALM experiments, oocytes were fixed with $1 \%$ paraformaldehyde (PFA) for 30 minutes after removal of the vitelline envelope and prior to imaging. This light fixation did not completely stop GlyR transport from the endoplasmic reticulum (ER), however higher PFA concentrations or longer incubation times with PFA lead to a decreased integrity of the oocyte and crosslinking of multiple GlyR channels and therefore could not be used. The imaging was carried out in the ND96 solution, at $\mathrm{pH} 7.5$ and at room temperature unless otherwise stated in the text.

\section{Single step photobleaching and single molecule counting-PALM experiments}

Images were acquired on a custom-built total internal reflection fluorescence (TIRF) microscope based on an Olympus IX71 inverted microscope frame fitted with a 100X 1.4 NA oil immersion objective. Excitation light at $488 \mathrm{~nm}$ from an Argon-Krypton laser (Coherent Inc) was used to excite the native mEos2, mEos3.1, mEos 3.2, mClavGR2, mMaple and Dendra2 as well as the photoactivated form of PA-GFP in TIRF mode. Excitation light at 560

$\mathrm{nm}$ from a fiber laser (MPB Communications) was used to excite the photoactivated form of PA-mCherry or the photoconverted form of mEos2, mEos3.1, mEos3.2, mClavGR2, mMaple 
and Dendra2 in TIRF mode. Excitation light from a $405 \mathrm{~nm}$ solid state laser (Cube, Coherent Inc) was used to photoactivate all eight fluorescent proteins in TIRF mode. The emission of native mEos2, mEos3.1, mEos3.2, mClavGR2, mMaple, Dendra2 and photoactivated PAGFP was collected with a BP 525/50 emission filter (Chroma Technology) and that of photoconverted mEos2, mEos3.1, mEos3.2, mClavGR2, mMaple, Dendra2 and photoactivated PA-mCherry was collected with BP 605/52 (Chroma Technology). The fluorescence emission was recorded onto an EMCCD camera (pixel size $157 \mathrm{~nm}$ ) (Andor Technology) with an exposure time of $100 \mathrm{~ms}$ per frame for single step photobleaching experiments and $50 \mathrm{~ms}$ per frame for single molecule counting-PALM experiments. For single step photobleaching experiments, fluorescent proteins were photoactivated (or photoconverted) by exposing them to the photoactivation laser at varying powers and durations (as indicated in the manuscript). The single step photobleaching was then achieved by exposing PA-GFP to $488 \mathrm{~nm}$ laser light at $7.8 \mathrm{~W} / \mathrm{cm}^{2}$, PA-mCherry to $560 \mathrm{~nm}$ laser light at $9.7 \mathrm{~W} / \mathrm{cm}^{2}$, mEos 2 to $488 \mathrm{~nm}$ or $560 \mathrm{~nm}$ laser light at $4 \mathrm{~W} / \mathrm{cm}^{2}$ and $9.7 \mathrm{~W} / \mathrm{cm}^{2}, \mathrm{mEos} 3.1$ and mEos 3.2 to $488 \mathrm{~nm}$ or $560 \mathrm{~nm}$ laser light at $11 \mathrm{~W} / \mathrm{cm}^{2}$, mClavGR2 to $488 \mathrm{~nm}$ or $560 \mathrm{~nm}$ laser light at $11 \mathrm{~W} / \mathrm{cm}^{2}$ and $16 \mathrm{~W} / \mathrm{cm}^{2}$, mMaple to $488 \mathrm{~nm}$ or $560 \mathrm{~nm}$ laser light at $5.5 \mathrm{~W} / \mathrm{cm}^{2}$ and $13 \mathrm{~W} / \mathrm{cm}^{2}$, and finally Dendra2 to $488 \mathrm{~nm}$ or $560 \mathrm{~nm}$ laser light at $4 \mathrm{~W} / \mathrm{cm}^{2}$ and 17 $\mathrm{W} / \mathrm{cm}^{2}$. For single molecule counting-PALM, mEos2, mEos3.2, mClavGR2, mMaple and PA-mCherry were photoactivated over time by illuminating the sample with $405 \mathrm{~nm}$ laser for $50 \mathrm{~ms}$ every $0.5 \mathrm{~s}$. For mEos 2 , mEos3.2, mClavGR2, mMaple and PA-mCherry, the laser power was increased in small increments every $10 \mathrm{~s}$ and followed a sigmoidal rise during the first 6 min until the power reached $9.5 \mathrm{~W} / \mathrm{cm}^{2}$. The $405 \mathrm{~nm}$ power was then kept constant until the end of the measurement. Dendra2 was photoactivated following the same procedure, except the maximum $405 \mathrm{~nm}$ power was lowered to $5.4 \mathrm{~W} / \mathrm{cm}^{2}$. These fluorescent proteins were simultaneously imaged and photobleached by exposure to $560 \mathrm{~nm}$ laser at $1 \mathrm{~kW} / \mathrm{cm}^{2}$. PA-GFP was simultaneously photoactivated, imaged and photobleached by exposure to 488 $\mathrm{nm}$ laser at $162 \mathrm{~W} / \mathrm{cm}^{2}$. Data acquisition in PALM experiments typically lasted for $\sim 10 \mathrm{~min}$.

\section{Data Analysis:}

\section{Single step photobleaching}

A square region of $3 \times 3$ pixels around the center of each spot was chosen to extract intensitytime traces using custom written MatLab software ${ }^{43}$. Background was subtracted locally by considering the average intensity of a $2 \times 2$ pixel region surrounding the molecule of interest. We excluded from our analysis: spots that moved by more than one pixel before they photobleached, spots that were fluorescent for only 1 frame and multiple partially overlapping spots. The steps in intensity-time traces were counted manually. Photobleaching steps were identified manually and defined as events in which the mean intensity of the smallest step was at least twice the standard deviation of the background fluorescence. We corrected for missed events in which both fluorescent proteins photobleached simultaneously as previously described ${ }^{36}$. Briefly, we calculated the photon distribution of single fluorescent proteins from traces that contained two photobleaching steps and characterized traces containing a single step size larger than twice the mean of this distribution as missed events. To determine the reliability of manual counting and the number of traces needed for a confident determination of the photoactivation efficiency, we used computer simulated traces 
taking into account the fluorescent protein photophysics such as signal to noise ratio, blinking and photobleaching kinetics (see below and Supplementary Fig. 12 for more details).

When the subunit stoichiometry of the receptor is constant, the observed distribution of steps should follow the binomial distribution given by:

$$
q_{n}=\left(\frac{M !}{n !(M-n) !}\right) P_{f}^{n}\left(1-P_{f}\right)^{M-n}
$$

Where, $q_{n}$ is the probability to observe $n$ steps in a time trace, $M$ is the total number of subunits, $n$ is the observed number of steps, $P_{f}$ is the probability for the fluorescent protein to be fluorescent (or photoactivation efficiency in the text). For two subunits the above expression reduces to a series of three equations: $q_{2}=P_{f}^{2}$ (probability for two steps), $q_{1}=2$ $P_{f}\left(1-P_{f}\right)$ (probability for one step) and $q_{0}=\left(1-P_{f}\right)^{2}$ (probability for completely dark channels). The probability, $P_{f}$, for the fluorescent protein to be fluorescent can be estimated by directly fitting the observed number of steps to the above equation.

\section{Single molecule counting-PALM}

PALM traces were extracted in the same way as the single step photobleaching traces. A threshold for the average intensity was set as 4-6 times the standard deviation of the background fluorescence and events in the traces that were above this threshold were identified as peaks. Typically each peak lasted for one or two frames. The fluorescence offtimes were determined as the time after the intensity of one peak decayed to the background level and the next peak appeared above the intensity threshold. Fluorescence off-times were fit to an exponential and a cut-off time was set as the time in which the exponential decayed to $99.8 \%$ of its initial value. Peaks with off-times shorter than or equal to this cut-off time were then linked together.

In a few cases the oocyte membrane disintegrated due to increased sensitivity to proteinase $\mathrm{K}$ digestion as evidenced by highly fluorescent background appearing as the yolk leaked out. These oocytes were not used for imaging or data analysis. Finally, in a few cases the photoactivation during PALM imaging completely failed as evidenced by very low overlap of detected spots in PALM with the mCherry image as well as very low coincidence between the activating light pulse and the appearance time of fluorescence spots in the PALM movie. These samples were excluded from the analysis.

\section{Simulations of photobleaching traces}

A Monte Carlo method was used for simulating traces as follows. The fluorescence signal from all fluorophores was calculated as a function of time and as time was incremented a comparison was made between a random number and a simulation parameter to determine if a certain event happened. The simulation parameters that we used for the comparison were the probability for the fluorescent protein to be on, $P_{f}$, the photobleaching lifetime, $\tau_{\text {bleach }}$, and the blinking lifetime, $\tau_{\text {blink }}$ for which a molecule was either in the "on" or "off" state. At time equal to zero, a random number was generated from a uniform distribution and if this random number was less than $P_{f}$ then the fluorophore was set to be in a bright state; otherwise the fluorophore remained in the dark state for the entire trace. To simulate a bleaching event, a random number was generated from an exponential distribution, with lifetime $\tau_{\text {bleach, }}$ and was 
defined to be the time at which bleaching occurred. At each time increment in the simulation a comparison was made between the value of the simulation time and the value of the randomly-generated bleaching time and if the simulation time was greater than the bleaching time then the fluorophore photobleached. Similarly, to simulate blinking, a random number was generated from an exponential distribution, with lifetime $\tau_{\text {blink, }}$, and was defined as the duration for which the fluorophore was in either a bright or a dark state. To determine which state the fluorophore was in, another random number was generated from a uniform distribution and if this random number was greater than 0.5 then the fluorophore was in a bright state; otherwise it was in a dark state. When the simulation time progressed to the end of this blinking event another randomly-generated blinking event was obtained and these blinking events repeated until the simulation time reached the maximum time specified for the trace. The simulated signal from each fluorophore was multiplied by a constant value and random noise (from a Poisson distribution with a specified expectation value) was added to adjust the signal-to-noise ratio of the trace. Simulated traces were generated by one researcher and counted in a double blind way by another researcher.

\section{References:}

41. Goldin, A.L. Maintenance of Xenopus laevis and oocyte injection. Methods Enzymol 207, 266-279 (1992).

42. Wang, M.H. A technical consideration concerning the removal of oocyte vitelline membranes for patch clamp recording. Biochem Biophys Res Commun 324, 971-972 (2004).

43. Heyes, C.D., Kobitski, A.Y., Breus, V.V. \& Nienhaus, G.U. Effect of the shell on the blinking statistics of core-shell quantum dots: A single-particle fluorescence study. Phys. Rev. B 75, 125431-125439 (2007). 
a

Population of GyRs before photoactivation

$$
\begin{aligned}
& a^{\beta} a a^{\beta} a \\
& \text { (a) } \beta \\
& \alpha \beta \beta
\end{aligned}
$$

b
GyR before photoactivation
Sequential photoactivation imaging and photobleaching

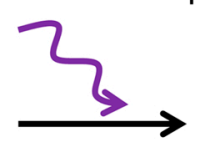

Population of GyRs after photoactivation

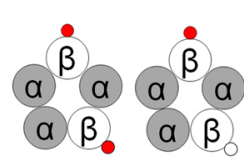

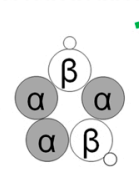

Intensity-time traces with steps
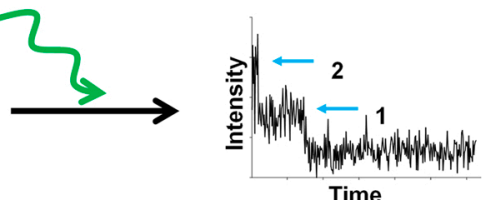

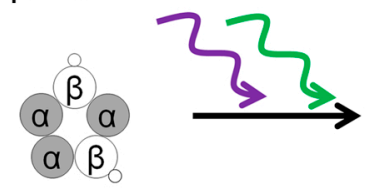

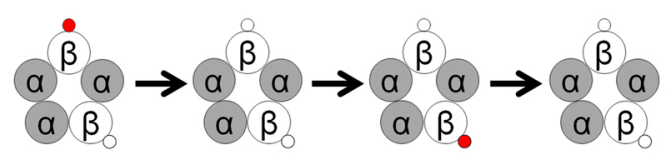

Intensity-time traces with peaks

a $\beta$

a) $\beta$

a $\beta$.

a $\beta$

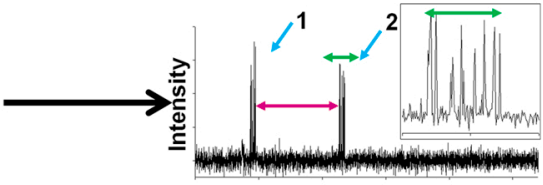

Time 

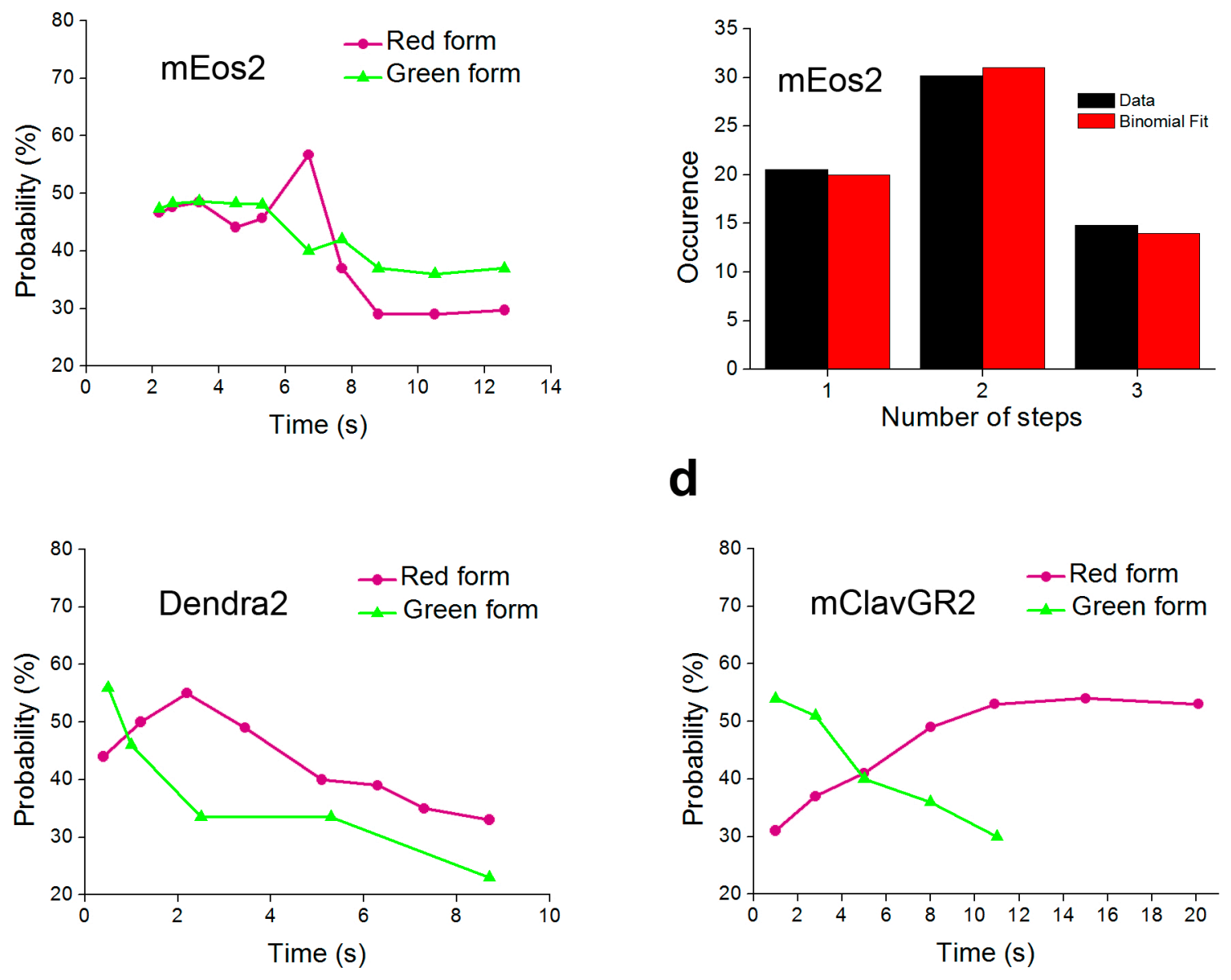
a

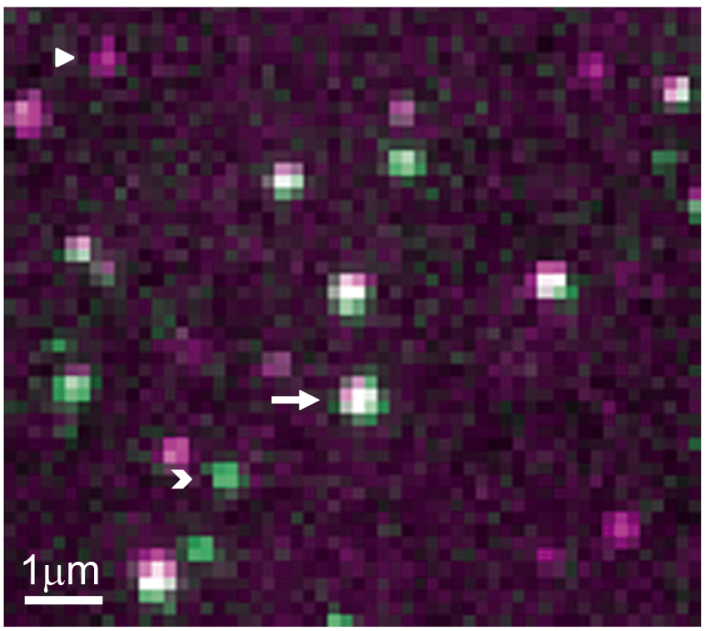

b

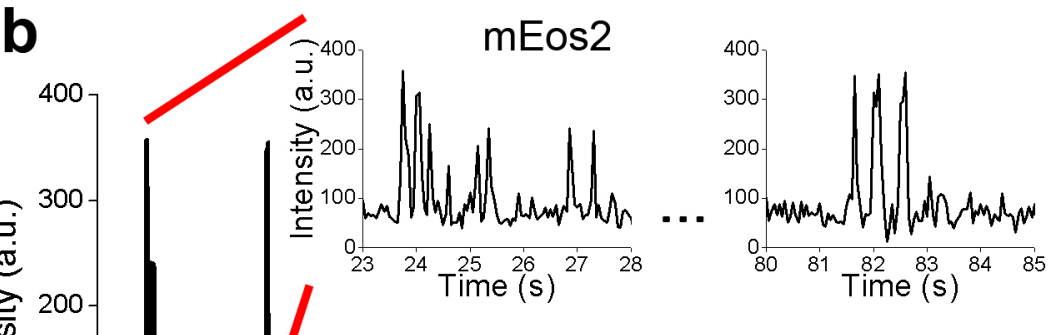

产

100
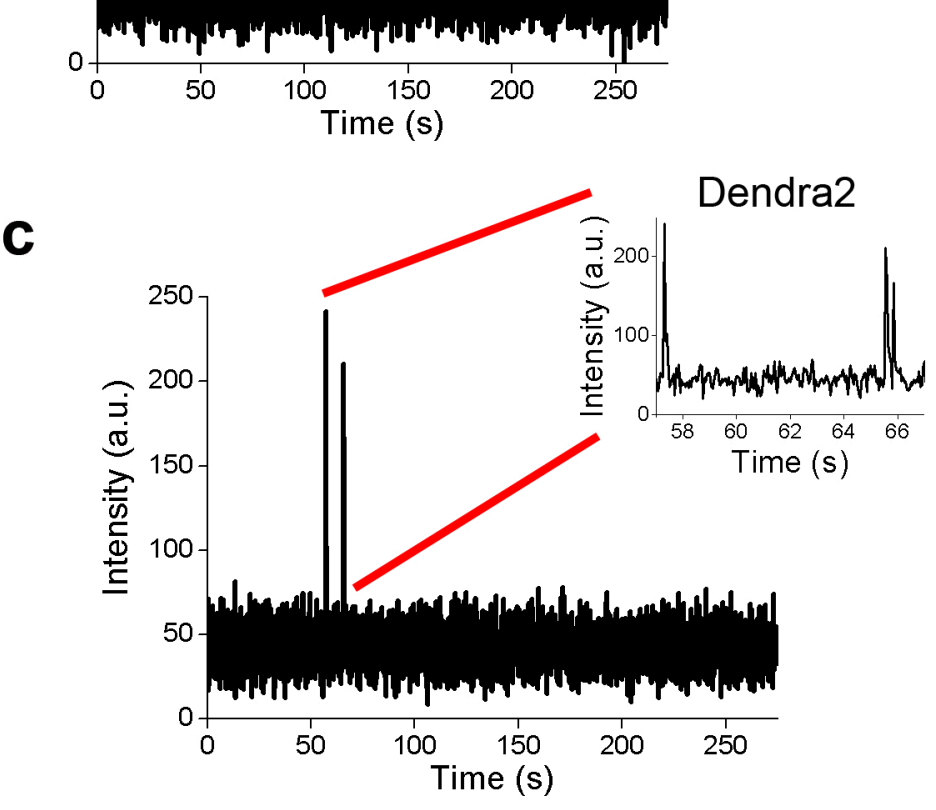
a

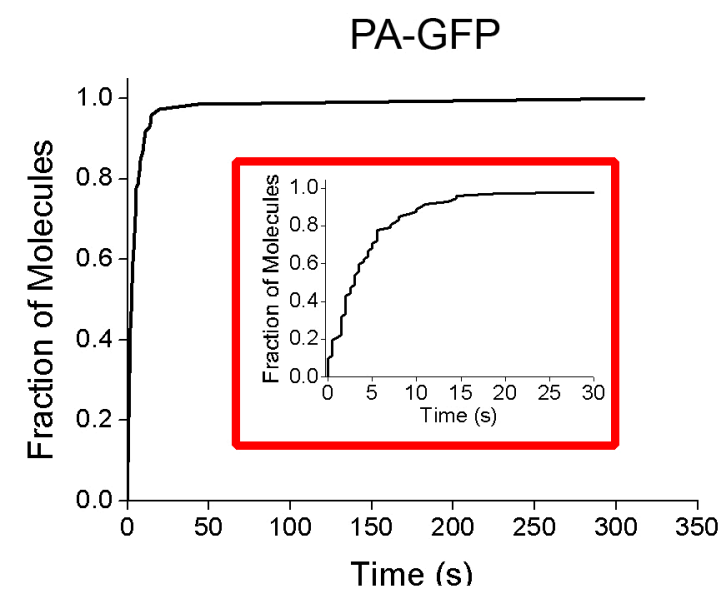

C

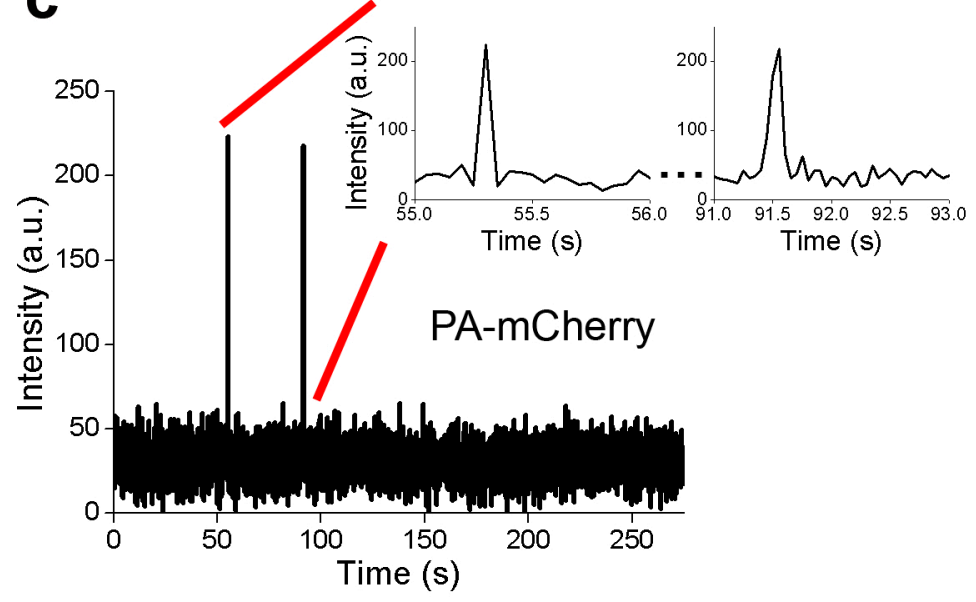

b
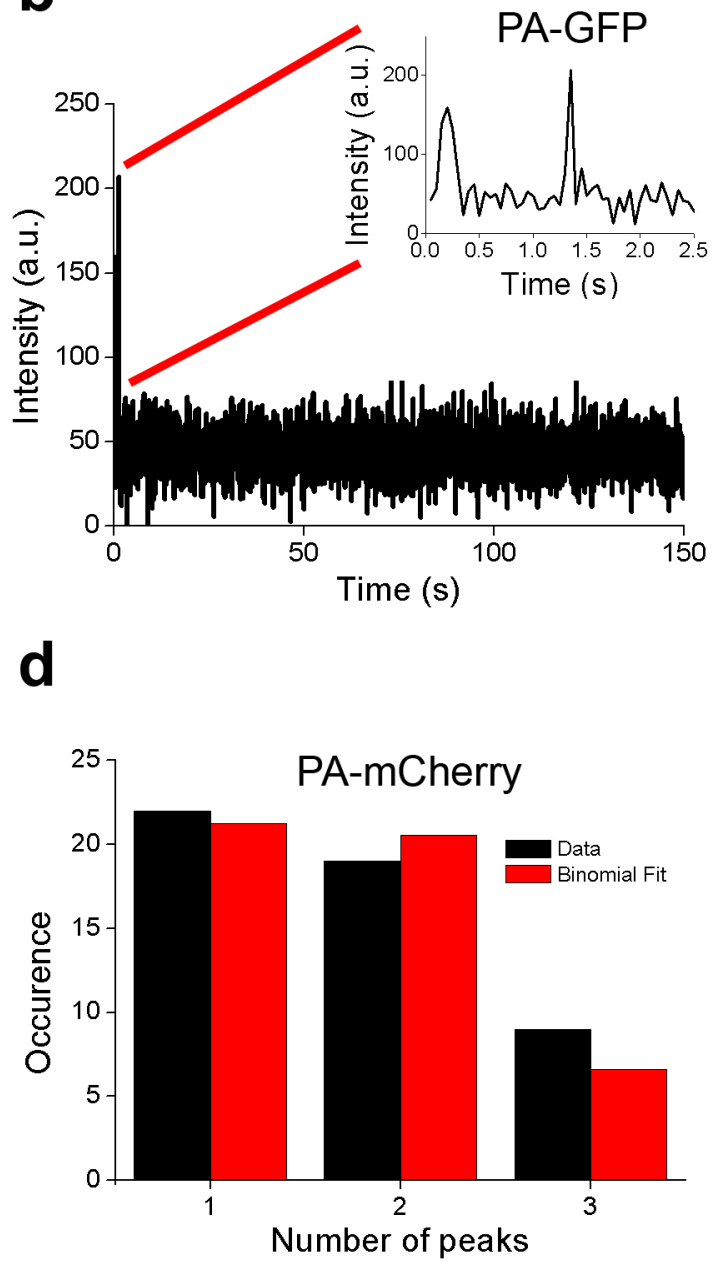